\title{
Alcohol Abuse and Youth: Relation between Mental Health and Their Adjustment.
}

\author{
Dr. Rajat Kumar Jain ${ }^{1}$, Dr. Gayatri Jay Mishra ${ }^{2}$, Dr.Vandna Singh ${ }^{3}$ \\ ${ }^{1}$ Reader In Education, ${ }^{2}$ Associate Professor In Education, ${ }^{3}$ Lecturer In Education \\ Department Of Education Shri Shankaracharya Mahavidyalya,
}

\begin{abstract}
Now a days world is becoming more and more comfortable. Everyone wants to have more of the comfort in his share by every possible way, especially youths are the pray of this these all. To get the desire comfort and happiness, they want to keep away all the difficulties and frustration from them and there is the easiest way for this is taking drug and Alcohol. But its affect their lives, families and societies and it becomes problems for them as well as people around them.

Aim of the study: The aim the present studies is to check the association between the mental health and their adjustment among the alcohol abusing students especially those who are living in rented room or hostels of Durg district.

Method: 100 male and female have been chosen for this studies from several colleges of Durg District especially the students doing graduation from different disciplines.

Result: It was found that there exists Positive relationship between Alcoholic Mental Health and their Adjustment. A Positive relationship has been found between Alcoholic Male youth Mental Health and their Adjustment and also Positive relationship between Alcoholic Female Mental Health and their Adjustment is found. There exists a significance difference between Alcoholic Male-Female Mental Health but there is no significance difference was found between Alcoholic Male-Female Adjustment.
\end{abstract}

\section{Introduction}

Happiness is essential for every human-being. For this purpose people made so many things which are good or bad. Alcohol is one of them Alcohol is the most commonly used drug in the world including in India. Alcohol has become an important part of our society and culture for many centuries. Sometime it is a symbol of joy. People of all ages across India have an intimate relationship with alcohol, with positive as well as negative effects in the short and longer term. Is it really joyful or not? Alcohol is tied up with many areas of our lives, and we use it in a plethora of ways: to help us relax, feel brave, introduce ourselves, seal business deals, celebrate life events, drown our sorrows, remember, forget, welcome people, say goodbye to people, get to know people, manipulate people, because we feel like it, because we need it, to numb ourselves, to feel grown up, to feel young, to belong, to distinguish ourselves, and sometimes, because we've forgotten how to do anything without alcohol. Over the past few years alcohol consumption has increased to the doubled compared to the previous decade in India. But it is a question arise Why a man really taking alcohol? It is a matter of discussion for a long time. Ramlan et. al. (2015) said that there are many reasons and factors that lead to the alcoholism and this issue will lead to the undesirable effects and impacts to an individual. It is a matter of too much attention.

Alcohol disturbed human's Mental Health and collapsed their Adjustment ability in society. Research findings shows that Indian youth die at much younger ages than the general population caused by kidney failure and its major cause is Alcohol. Mental Health and Adjustment are two factors which decide our status in society and friend circle. Coleman (1970) defined Mental Health as the ability to balance feelings, desires ambitions and ideas in one's daily livings and to face and accept the realities of life. It is the habit of work and attitude towards people and things that brings maximum satisfaction and happiness to the individuals. Where Dandapani (2000) is described that "Mental Health refers to the full and harmonious functioning of our total personality as well as to our bio-socio-psychological and spiritual well being". The term adjustment here refers to the adequacy of personal and interpersonal processes that we use to adapt to the environment such as reactions to stressors (Lazarus \& Cohen 1977). Hence adjustment is a condition of harmonious relationship between the social and physical environment wherein an individual is able to obtain optimum satisfaction and balance for significant needs and to meet the physical, mental and social demands imposed upon him. A welladjusted person engages in behaviors that are appropriate for the culture and given interpersonal situations to cope with life threats (Norris \&Kaniasty, 1996). It also means being flexible and subject to positive adaptability to environmental changes and stressors (Campbell, 1983). 


\section{Related Research}

1.David, John , Elizabeth et al(2005)Examined In a study the linkages between the extent of depressive symptoms (asymptomatic, sub threshold, major depression) among youth and mental health outcomes up to age 25 years in a New Zealand birth cohort. They found significant associations between the extent of depression at ages 17 to 18 years and rates of subsequent depressive symptoms, major depression, treatment for depression, anxiety disorder, and treatment for anxiety disorder, suicidal ideation, and suicide attempts. After adjustment for covariate factors, the extent of depression at ages 17 to 18 years remained associated with later depression and suicidal tendencies)

2. Harter (2000). This review examines controlled studies of the psychosocial adjustment of adult children of alcoholics (ACOAs) published since 1988. ACOAs appear at increased risk for a variety of negative outcomes, including substance abuse, antisocial or under controlled behaviors, depressive symptoms, anxiety disorders, and low self-esteem, difficulties in family relationships, and generalized distress and maladjustment. However, none of these outcomes are uniformly observed in ACOAs and none are specific to ACOAs. Comorbid parental pathology, childhood abuse, family dysfunction, and other childhood stressors may contribute to or produce similar outcomes.

3. Carlos, Mayumi and Crystal (2008). To assess the 12-month prevalence of psychiatric disorders, sociodemographic correlates, and rates of treatment among individuals attending college and their non-collegeattending peers in the United States. Results almost half of college-aged individuals had a psychiatric disorder in the past year. The overall rate of psychiatric disorders was not different between college-attending individuals and their non-college-attending peers. The unadjusted risk of alcohol use disorders was significantly greater for college students than for their non-college-attending peers, although not after adjusting for background socio demographic characteristics. College students were significantly less likely (unadjusted and adjusted) to have a diagnosis of drug use disorder or nicotine dependence or to have used tobacco than their non-college-attending peers. Bipolar disorder was less common in individuals attending college. College students were significantly less likely to receive past-year treatment for alcohol or drug use disorders than their non-college-attending peers.

4. Weinberger and Bartholomew (1996). In a research on Individual differences in social-emotional adjustment, jointly defined by levels of distress and self-restraint, were used to evaluate (a) patterns of alcohol use, (b) reasons for use, and (c) associated problems in two college samples of young adults. As hypothesized, low self-restraint was associated with high levels of alcohol use, drinking to increase positive effect, and high levels of alcohol-related problems. Subjective distress was not related to levels of use; however, it was associated with drinking to escape negative moods and social discomfort and with excessive alcohol-related problems. Both high distress and low self-restraint predicted problem drinking beyond what could be accounted for by quantity or frequency of alcohol use or by peers' use.

5. Turner, Larimer and Sarason, (2000). Studied the relationship between perceptions of parent-child conflict and alcohol-related consequences was examined in a sample of first-year fraternity and sorority members. Father-child conflict was a significantly better predictor for male students. Parent histories of problem drinking did not account for this relationship. Although male students reported substantially higher rates of drinking, the relationship between drinking and alcohol-related consequences was stronger among female students. Parentchild conflict at baseline also predicted adjustment to college at 1-year follow-up. Students who perceived higher levels of mother-child and father-child conflict reported higher levels of depression. Students who perceived higher levels of father-child conflict reported higher levels of global psychological distress. Conclusions: Results indicate that perceptions of conflict in specific parent-child relationships constitute a risk factor for poor college adjustment and the experience of alcohol-related consequences.

6. Kumar and Pathak (2014). In a research to study about mental health and social adjustment among college students. They found there is no significant Difference in mental health of college students' boys and girls. There is no significant difference in Social adjustment of college students boys and girls. The significant corelation between mental health and social adjustment reveals 0.69 high positive correlations.

These studies show that Mental Health and Adjustment of human being is very important. A lot of studies done by Researcher, Scientists, Economist, Psychologist etc. for awareness to people.

\section{Objectives of the Studies}

1. To examine the relationship between Alcoholic youth's Mental Health and their Adjustment.

2. To check the association of adolescent using alcohol and their mental health and adjustment.

3. To examine the difference between Alcoholic Male/Female Mental Health and their Adjustment.

\section{Hypothesis -}

1. There exists Positive relationship between Alcoholic youth's Mental Health and their Adjustment.

2. There exists Positive relationship between Alcoholic Male Mental Health and their Adjustment. 
3. There exists Positive relationship between Alcoholic Female Mental Health and their Adjustment.

4. There exists no significance difference between Alcoholic Male-Female Adjustment.

5. There exists no significance difference between Alcoholic Male-Female Mental Health.

Tool -

The investigators used the following standardized tools. "Mental Health Check-List" (MHC) constructed and standardized by Pramod Kumar. "Adjustment inventory For College Students" (AICS) constructed and standardized by A.K.P Sinha and R.P. Singh. This scale consists of 102 statements; each statement is in Yes or No type.

\section{Result And Analysis}

Table -1 H1. There exists Positive relationship between Alcoholic youth's Mental Health and their Adjustment.

\begin{tabular}{|c|l|c|c|c|c|}
\hline SN. & \multicolumn{1}{|c|}{ Variable } & $\mathrm{N}$ & Mean & SD & $\mathrm{r}$ \\
\hline 1. & Youth's Adjustment & 100 & 36.79 & 3.01 & 0.66 \\
\cline { 4 - 5 } 2. & $\begin{array}{l}\text { Youth's Mental } \\
\text { Health }\end{array}$ & & 35.69 & 1.86 & \\
\hline \multicolumn{2}{|c|}{$\mathrm{df}=99$} & \multicolumn{2}{c}{$\mathrm{P}<0.01$} \\
\hline
\end{tabular}

Goal of this research is to identify the score of Youth's adjustment and their mental health in which to find the ' $r$ ' value of Alcoholic students is ' $r$ ' is 0.66 which is significant at 0.01 level table value 0.254 mean wise we say that adjustment and mental health of youth strongly co- related to each other. Hence the hypothesis is accepted.

Table -2 H2. There exists Positive relationship between Alcoholic Male youth Mental Health and their Adjustment.

\begin{tabular}{|c|c|c|c|c|c|}
\hline SN. & Variable & N & Mean & SD & r \\
\hline 1. & Male Youth Adjustment & \multirow{2}{*}{50} & 36.98 & 3.62 & \multirow{2}{*}{0.913} \\
\cline { 1 - 2 } 2. & Male Youth Mental Health & & 34.74 & 1.44 & \\
\hline \multicolumn{2}{|c|}{$\mathrm{df}=49$} & $\mathrm{P}<0.01$ & \multicolumn{3}{|c|}{ Significant } \\
\hline
\end{tabular}

Goal of this research is to identify the score of male Youth adjustment and their mental health in which to find the ' $r$ ' value of Alcoholic students is ' $r$ ' is 0.913 which is significant at 0.01 level table value 0.354 mean wise we say that adjustment and mental health of youth strongly co-related to each other. Hence the hypothesis is accepted.

Table -3 H3. There exists Positive relationship between Alcoholic Female Mental Health and their Adjustment.

\begin{tabular}{|c|c|c|c|c|c|}
\hline SN. & Variable & $\mathrm{N}$ & Mean & SD & $\mathrm{r}$ \\
\hline 1. & Female Youth Adjustment & \multirow{2}{*}{50} & 36.48 & 2.25 & \multirow{2}{*}{0.911} \\
\hline 2. & Female Youth Mental Health & & 36.66 & 1.73 & \\
\hline \multicolumn{2}{|c|}{$\mathrm{df}=49$} & \multicolumn{2}{|c|}{$\mathrm{P}<0.01$} & \multicolumn{2}{|c|}{ Significant } \\
\hline
\end{tabular}

Goal of this research is to identify the score of male Youth adjustment and their mental health in which to find the ' $r$ ' value of Alcoholic students is ' $r$ ' is 0.911 which is significant at 0.01 level table value 0.354 mean wise we say that adjustment and mental health of youth strongly co-related to each other. Hence the hypothesis is accepted.

Table -4 H4. There exists no significance difference between Alcoholic Male Female Adjustment.

\begin{tabular}{|c|c|c|c|c|c|}
\hline SN. & Variable & N & Mean & SD & t \\
\hline 1. & Male Adjustment & 50 & 36.98 & 3.62 & 0.627 \\
\hline 2. & Female Adjustment & 50 & 36.60 & 2.27 & Non \\
\hline \multicolumn{7}{|c|}{ Significant } \\
\hline
\end{tabular}

Goal of this research is to identify the score of male female adjustment in which to find the ' $t$ ' value of Alcoholic students is ' $t$ ' is 0.627 which is non-significant at 0.01 level table value 2.626 mean wise we say that adjustment male and female of youth does not differ to each other. Hence the hypothesis is accepted. 
Table -5

H5. There exists no significance difference between Alcoholic Male Female Mental Health.

\begin{tabular}{|c|c|c|c|c|c|}
\hline SN. & Variable & N & Mean & SD & t \\
\hline 1. & Male Mental Health & 50 & 34.74 & 1.44 & 5.88 \\
\hline 2. & Female Mental Health & 50 & 36.64 & 1.77 & \\
\hline \multicolumn{5}{|c|}{$\mathrm{P}<0.01$} & Significant \\
\hline
\end{tabular}

Our goal of this research is to identify the score of male female adjustment in which to find the ' $t$ ' value of Alcoholic students is ' $t$ ' is 5.88 which is significant at 0.01 level table value 2.626 mean wise we say that mental health of male and female is significant differ to each other. Hence the hypothesis is rejected.

\section{Interpretation -}

Our study indicates that alcohol plays a strong role of our life. We can't ignore it, while us treating the youth. Alcohol developed many dangerous symptoms like depression, loneliness and anti-social activities. It is very much responsible for their ill mental health and male Adjustment. If we want to remove these causes which can attract youth lust for alcohol, it is necessary for us o talk to youths, counselle them, aware to stop the wrong habit of youth. Teacher should give him focus and plan for their good health and open the door for their creativity. Teachers should give them good and quality education which developed their self control.

Many of the drawbacks of alcohol addiction. Heavy drinking can lead to inflammation of the liver, known as alcoholic hepatitis, and cirrhosis, or scarring of the liver. It also increases blood pressure, can damage the heart muscle and potentially which plays an important role in the development of several cancers, including breast, colon, mouth and liver. In addition to it, heavy drinking increases the risk for osteoporosis, particularly in young women, alcohol can disrupt sleep patterns and lead to weight gain. Drinking can also cloud your judgment, paving the way for destructive decisions such as getting behind the wheel of a car. The rates of high blood pressure increase and you're more likely to have a stroke.

\section{Reference}

[1]. Coleman, J. C., (1970), “Abnormal Psychology and Modern Life.”Taraporevala Sons \& Co. Pvt. Ltd., Bombay.

[2]. Dandapani (2000). A Text Book of Advanced Psychology. Anmol Publication Pvt. Ltd. New Delhi, India, pp. 510.

[3]. Campbell, (2006). Environmental Changes \& Stressors. New York London: psychology press, Taylor \& Francis Group.

[4]. Fergusson, David M., Horwood, L. John, Ridder, Elizabeth M. et al, (2005). Subthreshold Depression in Adolescence and Mental Health Outcomes in Adulthood, Arch Gen Psychiatry. 62(1):66-72. doi: 10.1001

[5]. Harter, Stephanie Lewis (2000). Psychosocial adjustment of adult children of alcoholics: A review of the recent empirical literature, Clinical Psychology Review, Volume 20, Issue-3, Pages 311-337)

[6]. Blanco, Carlos, Okuda, Mayumi, Crystal Wright et al (2008). Mental Health of College Students and Their Non-College-Attending Peers, Arch Gen Psychiatry. 2008; 65(12), pp.1429-1437.

[7]. A. Daniel, Weinberger, Bartholomew, Kim (1996). Social-Emotional Adjustment and Patterns of Alcohol Use among Young Adults, Journal of Personality, Volume 64, Issue 2, Pages 495-527

[8]. Turner, A P, Larimer, M E, Sarason, I G (2000). Family risk factors for alcohol-related consequences and poor adjustment in fraternity and sorority members: exploring the role of parent-child conflict, Journal of Studies on Alcohol, 61(6), 818-826.

[9]. Kumar, Yogesh and Pathak, V. (2014). Mental Health and Social Adjustment among College Students, International Journal of Public Mental Health and Neurosciences, Volume 1, Issue 1, p-11-14 